\title{
Temperature dependent local atomic displacements in Ru substituted $\mathrm{SmFe}_{1-x} \mathrm{Ru}_{x} \mathrm{AsO}_{0.85} \mathrm{~F}_{0.15}$ superconductors
}

\author{
Boby Joseph, ${ }^{1}$ Antonella Iadecola, ${ }^{1,2}$ Laura Simonelli, ${ }^{3}$ Laura Maugeri, ${ }^{1}$ \\ Alberto Martinelli, ${ }^{4}$ Andrea Palenzona, ${ }^{4}$ Marina Putti, ${ }^{4}$ and Naurang L. Saini ${ }^{1}$ \\ ${ }^{1}$ Dipartimento di Fisica, Università di Roma "La Sapienza", P. le Aldo Moro 2, 00185 Roma, Italy \\ ${ }^{2}$ Elettra, Sincrotrone Trieste, Strada Statale 14, Km 163.5, Basovizza, Trieste, Italy \\ ${ }^{3}$ European Synchrotron Radiation Facility, 6 RUE Jules Horowitz BP 22038043 Grenoble Cedex 9 France \\ ${ }^{4}$ CNR-SPIN and Università di Genova, via Dodecaneso 33, 16146 Genova, Italy
}

Local structure of $\mathrm{SmFe}_{1-x} \mathrm{Ru}_{x} \mathrm{AsO}_{0.85} \mathrm{~F}_{0.15}(x=0.0,0.05,0.25$ and 0.5$)$ superconductors has been investigated by temperature dependent As $K$-edge extended x-ray absorption fine structure. The effect of $\mathrm{Ru}$ substitution remains confined to the iron-arsenide layer but neither the static disorder nor the Fe-As bond strength suffers any change for $x \leq 0.25$. With further Ru substitution the static disorder increases while the Fe-As bond strength remains unchanged. Also, the Ru-As distance $(\sim 2.42 \AA)$, different from the Fe-As distance $(\sim 2.39 \AA)$, does not show any change in its force constant with the $\mathrm{Ru}$ substitution. These observations suggest that the $\mathrm{SmFe}_{1-x} \mathrm{Ru}_{x} \mathrm{AsO}_{0.85} \mathrm{~F}_{0.15}$ system breaks down to coexisting local electronic phases on isoelectric substitution in the active FeAs layer.

Journal reference : Supercond. Sci. Technol. 26 (2013) 065005

DOI: $10.1088 / 0953-2048 / 26 / 6 / 065005$

PACS numbers:

\section{INTRODUCTION}

The newly discovered iron-based high $T_{c}$ superconductors [1-3] have a particular layered structure with electronically active $\mathrm{FePn} / \mathrm{Ch}(\mathrm{Pn}=$ pnictogen; $\mathrm{Ch}=$ chalcogen $)$ layers alternated by spacer layers. The superconductivity and magnetism in these materials are strongly dependent on the thickness of the active layers (e.g., the height of $\mathrm{Pn} / \mathrm{Ch}$ atoms from the Fe-plane) [3]. Among the iron-based superconductors, the REFeAsO (RE=rare earth), the socalled 1111 system with well defined iron-arsenide active layers stacked together with the spacer layers shows the highest $T_{c}[2,3]$. Generally, atomic substitution in either of the stacking layers is used to control and manipulate superconductivity and other transport properties (e.g., a partial substitution of O by F) [3]. In addition to the control over the superconductivity, atomic substitution in the active layer also permits to understand the transport phenomena $[4,5]$ and develop new structures through a detailed information on the role of different layers. In particular, it is important to have knowledge of the local atomic correlations and modification introduced by the substituted atoms in the layered structure topology.

X-ray absorption spectroscopy is an atomic site-specific experimental probe [6], that does not require any long range crystal symmetry, and hence permits to have a direct access to the local atomic correlations. Indeed, x-ray absorption fine structure (EXAFS) and x-ray absorption near edge structure (XANES) measurements have been widely exploited to study the layered high $\mathrm{T}_{c}$ superconductots [7-10], including the iron based superconductors [11-19]. Earlier, we 
have explored the effect of different spacer layers (RE of different size) in the 1111-system [13, 14], and found that the interlayer atomic order/disorder should be important in these materials. We have also studied the effect of charge density varied by a partial substitution in the REO spacer layers ( $\mathrm{O}$ by $\mathrm{F}$ ), revealing key information on the interlayer atomic correlations and dynamics [16]. Recently we have focussed on the effect of isovalent Ru (atomic radius $1.34 \AA$ ) substitution directly in the active FeAs layer in place of Fe (atomic radius 1.26 $\AA$ ) of an optimally doped $\mathrm{SmFe}_{1-x} \mathrm{Ru}_{x} \mathrm{AsO}_{0.85} \mathrm{~F}_{0.15}$ [19]. The results have revealed that the local disorder induced by the Ru substitution is mainly confined to the FeAs layers which are getting thinner and decoupled from the SmO spacer layers. The present work is dedicated to distinctly identify the random static disorder and the bondlength fluctuations induced by an isoelectric substitution in the iron-arsenide active layer. For the purpose, we have performed temperature dependent As $K$-edge EXAFS measurements on a series of $\mathrm{SmFe}_{1-x} \mathrm{Ru}_{x} \mathrm{AsO}_{0.85} \mathrm{~F}_{0.15}(x=0.0,0.05,0.25$ and 0.5$)$ samples. Consistent with earlier work [19], the Ru substituted system is found to be characterized by different Fe-As and RuAs distances. Incidentally, the force constants for these distances remain unchanged with increasing Ru substitution, indicating distinct local electronic phases coexisting in the system. It appears that the system breaks down to coexisting nanoscale electronic phases due to isoelectric substitution, having a direct influence on the fundamental electronic properties of these materials.

\section{EXPERIMENTAL DETAILS}

The As $K$-edge $(\mathrm{E}=11868 \mathrm{eV})$ x-ray absorption measurements on powder samples of $\mathrm{SmFe}_{1-x} \mathrm{Ru}_{x} \mathrm{AsO}_{0.85} \mathrm{~F}_{0.15}(x$ $=0.0,0.25$ and 0.5) were performed in transmission mode at the beamline BM26A [20] of the European Synchrotron Radiation Facility (ESRF), Grenoble. Measurements on the $x=0.05$ sample were carried out at the XAFS beamline of the ELETTRA, Trieste using similar experimental approach. Temperature dependent measurements were carried out between 10 to $300 \mathrm{~K}$. Several scans were acquired at each temperature to ensure the spectral reproducibility. The EXAFS oscillations were extracted using the standard procedure based on spline fit to the pre-edge subtracted absorption spectrum [6]. The superconducting transition temperatures $\left(T_{c}\right)$, determined by resistivity measurements, are $51 \mathrm{~K}, 43 \mathrm{~K}, 14 \mathrm{~K}$ and $8 \mathrm{~K}$ respectively for the samples with $x=0.0,0.05,0.25$ and 0.5. Details on the sample preparation and characterization for transport and structural properties are described elsewhere [21-24].

\section{RESULTS AND DISCUSSIONS}

Figure 1 shows $\mathrm{k}^{2}$ weighted arsenic $K$-edge EXAFS of $\mathrm{SmFe}_{1-x} \mathrm{Ru}_{x} \mathrm{AsO}_{0.85} \mathrm{~F}_{0.15} \quad(x=0.0,0.05,0.25$ and 0.5$)$ at several temperatures. The effect of temperature and $\mathrm{Ru}$ substitution is evident from the EXAFS oscillations. For example, temperature dependent damping of EXAFS signal can be seen for all the samples. Similarly, the effect of Ru substitution can be seen in the EXAFS oscillations (see, e.g., $\mathrm{k} \geq 6 \AA^{-1}$ ). These effects can be better appreciated in the Fourier transforms of the EXAFS, providing real space information on the partial atomic distribution around the As atoms.

Figure 2 shows the Fourier transform (FT) magnitudes, obtained using a Gaussian window (k-range of EXAFS is 3-18 $\AA^{-1}$ ). There are four Fe/Ru near neighbours of arsenic at a distance $\sim 2.4 \AA$ (the main peak at $\sim 2 \AA$ ). The next nearest neighbours of arsenic are $\mathrm{Sm}$ atoms at $\sim 3.3 \AA$ and $\mathrm{O} / \mathrm{F}$ atoms at $\sim 3.5 \AA$ follwed by the As atoms at 


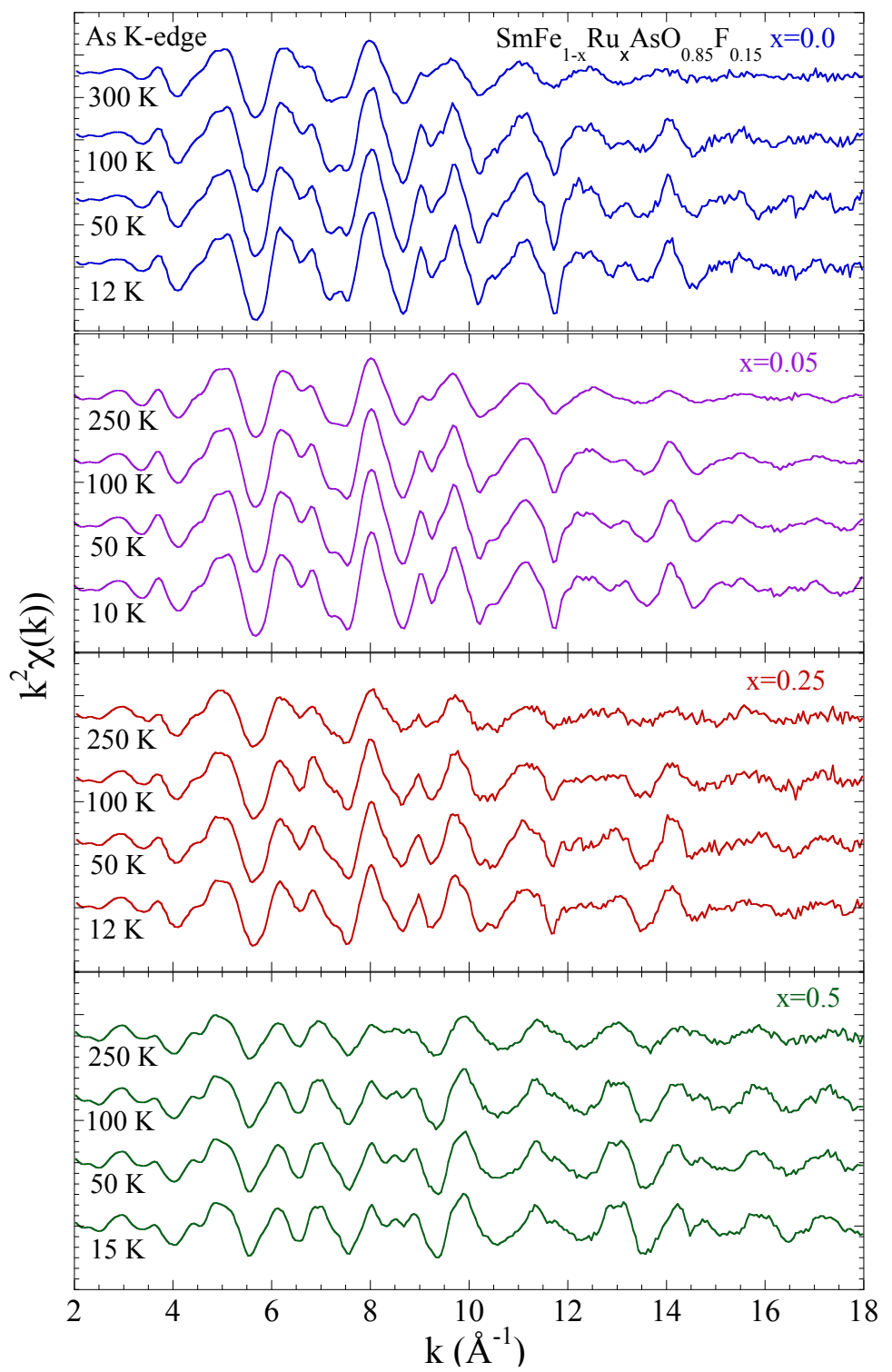

FIG. 1: Arsenic $K$-edge EXAFS of $\mathrm{SmFe}_{1-x} \mathrm{Ru}_{x} \mathrm{AsO}_{0.85} \mathrm{~F}_{0.15} \quad(x=0.0,0.05,0.25$ and 0.5$)$ at several temperatures (weighted with $\mathrm{k}^{2}$ ). The evolution of the local structure with temperature and Ru concentration is apparent from the EXAFS oscillations.

$\sim 3.9 \AA$ (the two peaks between 3-4 $\AA$ ), mixed with the multiple scattering contribution due to $\mathrm{Fe} / \mathrm{Ru}(\sim 4.6 \AA)$, appearing as FT peak at $\sim 4.2 \AA$ (see, e.g. top panel). It can be seen that the main FT peak is changing with Ru substitution while other FT peaks due to next near neighbour atoms suffering much smaller (or negligible) changes. Indeed, the main peak decreases substantially with $\mathrm{Ru}$ and appears as a clear doublet structure in the $x=0.5$ sample. Similarly, the multiple scattering Fe/Ru peak at $\sim 4.2 \AA$ sustains large change, having negligible weight in the $x=$ 0.5 sample. All these data are suggesting that the atomic disorder due to Ru substitution is mainly confined to the active layer, consistent with the earlier study [19]. On the other hand, the temperature dependent damping of the FT peaks appears almost similar in all the samples. 


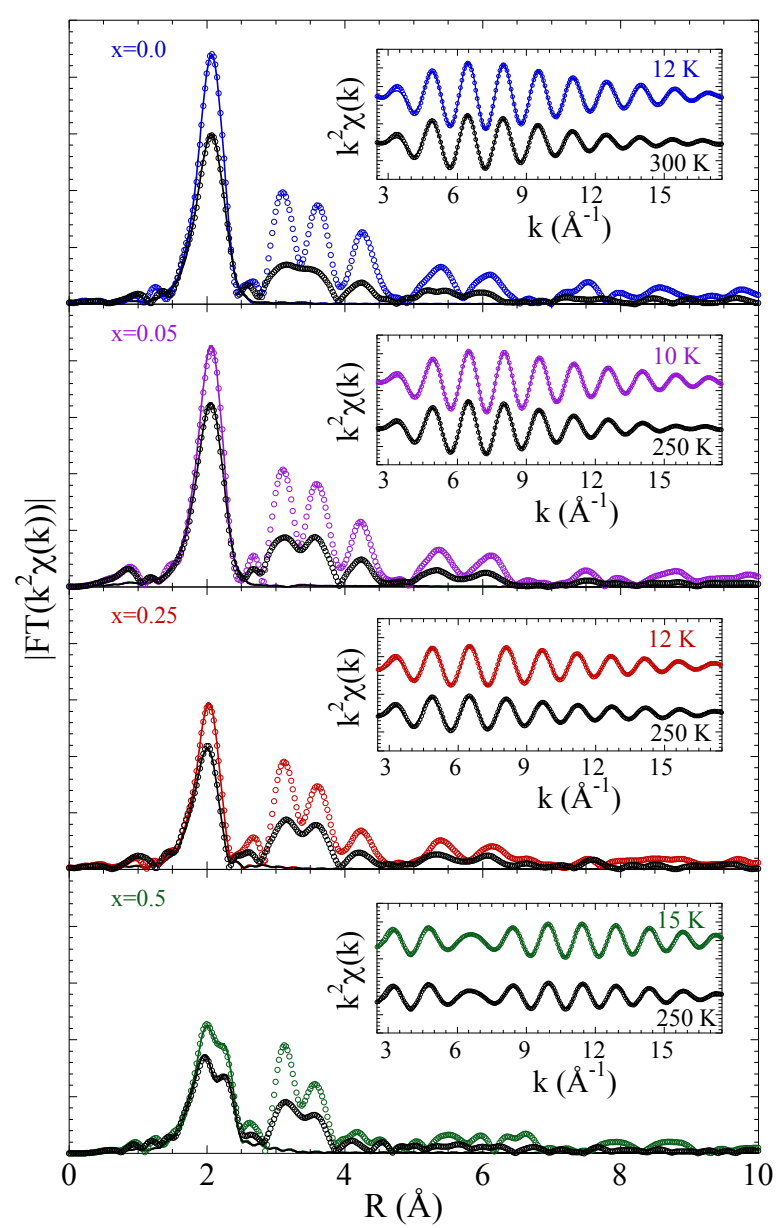

FIG. 2: Fourier transform magnitudes of the arsenic $K$-edge EXAFS at low and high temperatures (symbols) together with As$\mathrm{Fe} / \mathrm{As}-\mathrm{Ru}$ shells model fits (solid lines) for the $\mathrm{SmFe}_{1-x} \mathrm{Ru}_{x} \mathrm{AsO}_{0.85} \mathrm{~F}_{0.15}$ system. Insets show the filtered EXAFS oscillations (symbols) and the corresponding model fits (solid lines).

In the single-scattering approximation, the EXAFS is described by the following general equation[6]:

$$
\chi(k)=\sum_{i} \frac{N_{i} S_{0}^{2}}{k R_{i}^{2}} f_{i}\left(k, R_{i}\right) e^{-\frac{2 R_{i}}{\lambda}} e^{-2 k^{2} \sigma_{i}^{2}} \sin \left[2 k R_{i}+\delta_{i}(k)\right]
$$

where $\mathrm{N}_{i}$ is the number of neighbouring atoms at a distance $\mathrm{R}_{i}$ from the photoabsorbing atom. Here, $\mathrm{S}_{0}^{2}$ is the passive electrons amplitude reduction factor, $\mathrm{f}_{i}\left(\mathrm{k}, \mathrm{R}_{i}\right)$ is the backscattering amplitude, $\lambda$ is the photoelectron mean free path, $\delta_{i}$ is the phase shift, and $\sigma_{i}^{2}$ is the correlated Debye-Waller factor measuring the mean square relative displacement (MSRD) of the photoabsorber-backscatter pairs.

For the As $K$-edge EXAFS in the $\mathrm{SmFe}_{1-x} \mathrm{Ru}_{x} \mathrm{AsO}_{0.85} \mathrm{~F}_{0.15}$, the first shell contribution involves only the Fe-As/RuAs bonds, well separated from all other distant atom contributions $[11,12,16,19]$. To quantify the temperature dependent atomic displacements we have analyzed the EXAFS only due to the nearest neighbours. The filtered first shell EXAFS are displayed as insets of the Fig. 2. In the model fits we have varied the Fe-As/Ru-As distances and the corresponding $\sigma_{i}^{2}$, while other parameters including the photo-electron energy origin $\mathrm{E}_{0}$ (a value obtained by modeling five different scans at low temperature), the number of near neighbors $\mathrm{N}_{i}$ (an input from diffraction studies [21, 23]) and $\mathrm{S}_{0}^{2}(=1.0)$ were all kept fixed for the final iteration. Phase shifts and amplitude factors were calculated using the 


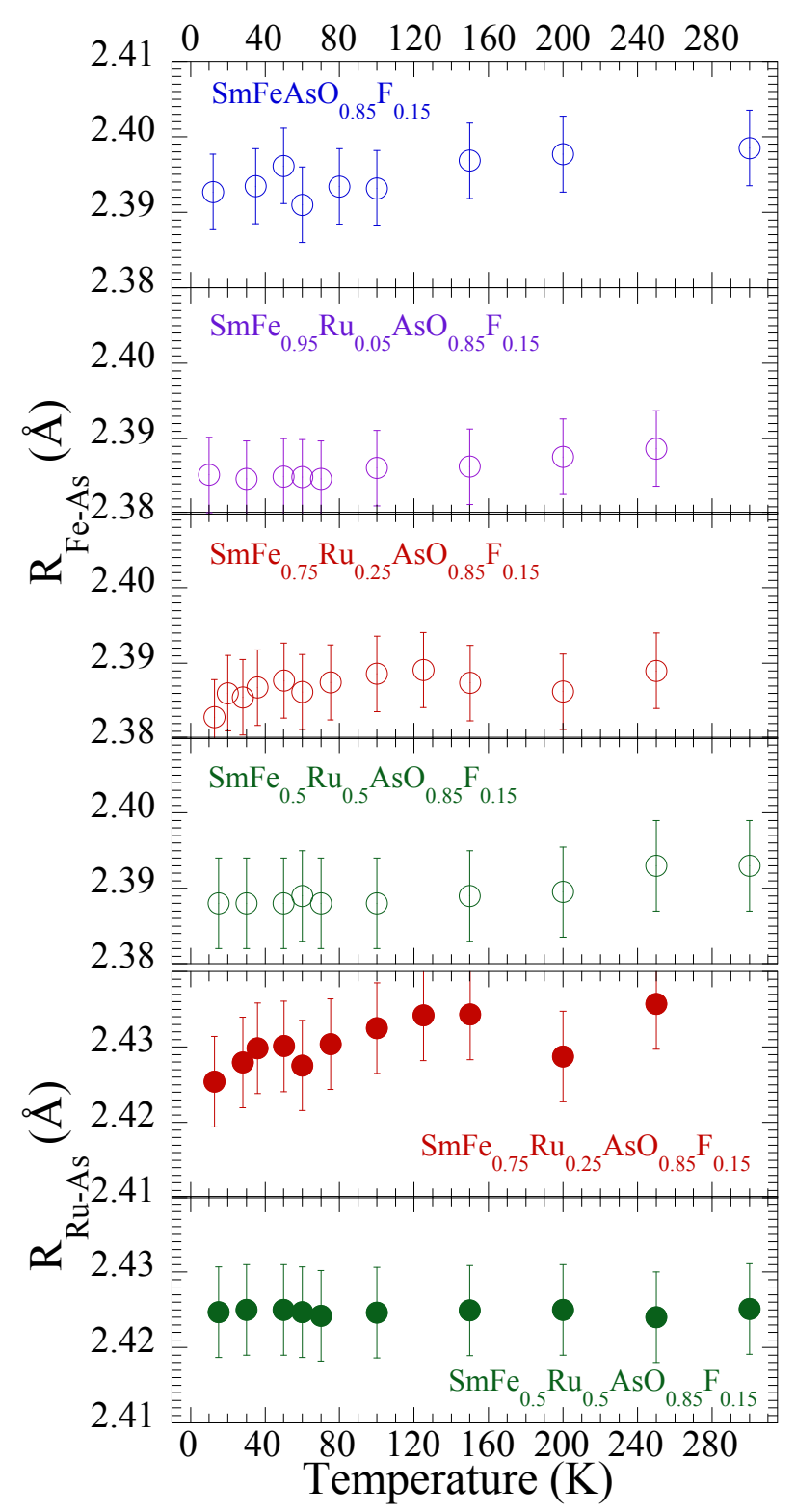

FIG. 3: Temperature dependence of Fe-As (upper four panels) and Ru-As (lower two panels) distances for the $\mathrm{SmFe}_{1-x} \mathrm{Ru}_{x} \mathrm{AsO}_{0.85} \mathrm{~F}_{0.15}(x=0.0,0.05,0.25$ and 0.5$)$ determined by As K-edge EXAFS analysis.

FEFF [25]. The number of independent data points for this analysis were about $11\left(\mathrm{~N}_{\text {ind }} \sim(2 \Delta \mathrm{k} \Delta \mathrm{R}) / \pi\right.$, where $\Delta \mathrm{k}$ $=15 \AA^{-1}$ and $\Delta \mathrm{R}=1.2 \AA$ are the $\mathrm{k}$ and $\mathrm{R}$ space over which the data have been analyzed) for a maximum of four parameters fits to the filtered EXAFS. The k-space (insets) and R-space model fits are also included in Fig. 2. The errors in the local structural parameters, determined by the EXAFS analysis, are estimated by creating correlation maps between different parameters and by analysis of different scans that are measured in the same conditions, following the known standards [26].

Figure 3 shows near neighbour (Fe-As and Ru-As) distances as a function of temperature, obtained from the EXAFS analysis. The related $\sigma_{i}^{2}$ are shown in Fig. 4. The Fe-As distance $(\sim 2.39 \AA)$ is different from the Ru-As distance $(\sim 2.42 \AA)$ with the difference $(\sim 0.03 \AA)$ being smaller than that measured in isostructural compounds like RuAs- 


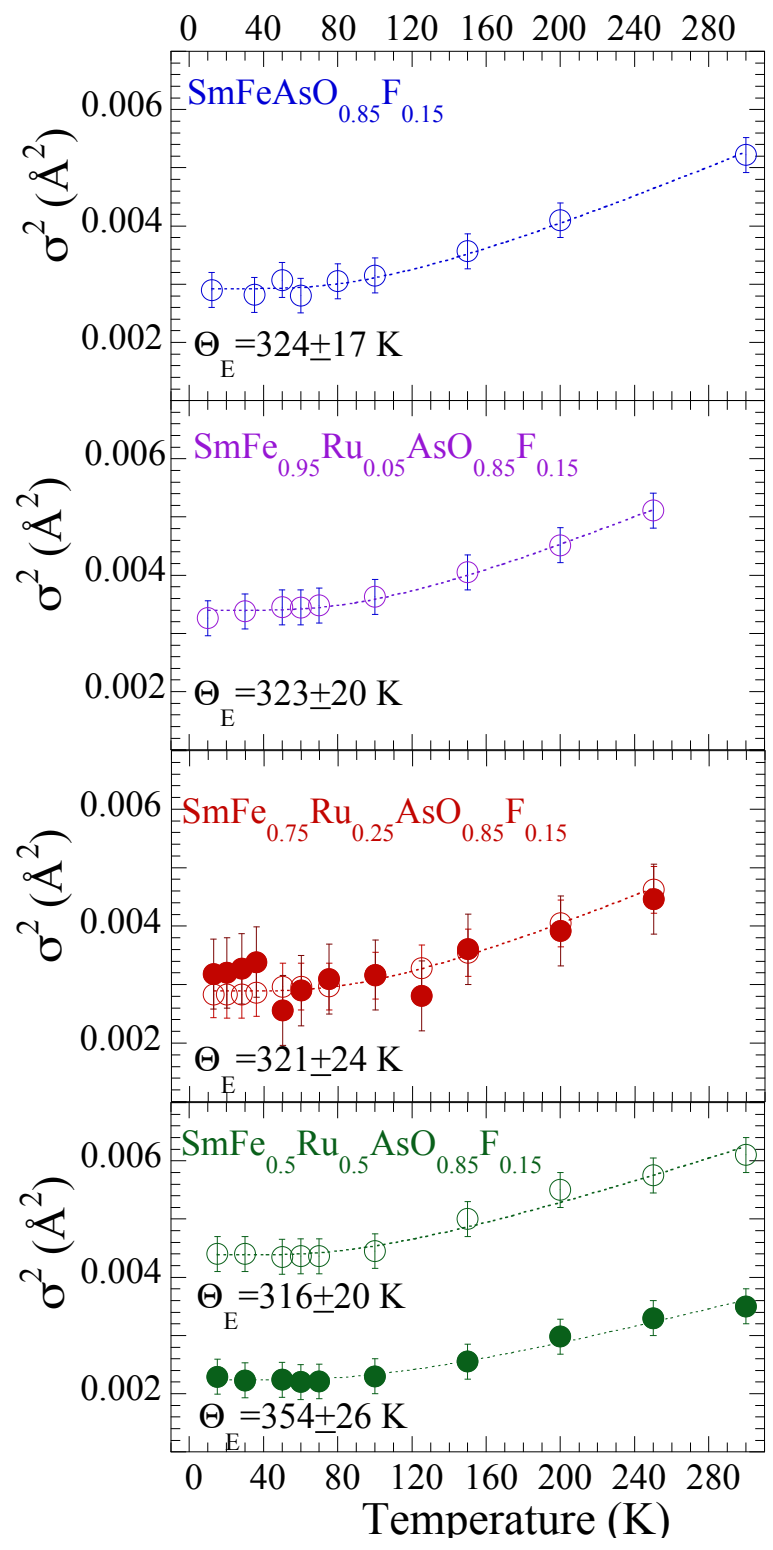

FIG. 4: Temperature dependence of mean square relative displacements in $\mathrm{SmFe}_{1-x} \mathrm{Ru}_{x} \mathrm{AsO}_{0.85} \mathrm{~F}_{0.15} \quad(x=0.0,0.05,0.25$, and 0.5) for the Fe-As (empty symbols) and Ru-As (filled symbols) bondlengths. The dotted lines are the correlated Einstein model fits.

FeAs and $\mathrm{RuAs}_{2}-\mathrm{FeAs}_{2}(\sim 0.06 \AA)$ [27]. This suggests the active layer in $\mathrm{SmFe}_{1-x} \mathrm{Ru}_{x} \mathrm{AsO}_{0.85} \mathrm{~F}_{0.15}$ is under chemical pressure from the REO spacter layer. Within the experimental uncertainties the local Fe-As (and Ru-As) distance remains constant with temperature and $\mathrm{Ru}$ concentration, consistent with strongly covalent nature of the Fe-As (and Ru-As) bonds that is known from earlier studies [13]. It is worth recalling that the reported error bars represent the maximum uncertainty (that is much higher than actual errors in most of the data points), that are determined by considering correlation between the fit parameters, i.e. the distances $\mathrm{R}_{i}$ and the $\sigma_{i}^{2}$.

The temperature dependence of the $\sigma_{i}^{2}$ permits to distinctly identify the random static disorder and the dynamic atomic displacements. The EXAFS $\sigma_{i}^{2}$ is a sum of temperature independent $\left(\sigma_{0}^{2}\right)$ and temperature dependent $\sigma_{i}^{2}(\mathrm{~T})$ 
terms [6], i.e.,

$$
\sigma_{i}^{2}=\sigma_{0}^{2}+\sigma_{i}^{2}(T)
$$

The temperature dependent term can be described by the correlated Einstein-model [28, 29],

$$
\sigma_{i}^{2}(T)=\frac{\hbar}{2 \mu \omega_{E}} \operatorname{coth}\left(\frac{\hbar \omega_{E}}{2 k_{B} T}\right),
$$

where $\mu$ is the reduced mass and $\omega_{E}$ is the Einstein-frequency of the pair of atoms involved (i.e.,Fe-As and Ru-As bonds). The related Einstein-temperature is $\Theta_{E}=\hbar \omega_{E} / k_{B}$. The fits to the correlated Einstein model are shown as dotted lines in Fig. 4. The $\Theta_{E}$ for the Fe-As bonds are found to be $324 \pm 17 \mathrm{~K}, 323 \pm 20 \mathrm{~K}, 321 \pm 24 \mathrm{~K}$ and $316 \pm 20 \mathrm{~K}$ for the $x=0.0,0.05,0.25$ and 0.5 samples respectively. The optical phonon modes in $\mathrm{SmFeAsO}^{\mathrm{are}} 201 \mathrm{~cm}^{-1}\left(\mathrm{~A}_{1 g}\right.$ involving As atom displacements) and $208 \mathrm{~cm}^{-1}\left(\mathrm{~B}_{1 \mathrm{~g}}\right.$ involving Fe atom displacements) [30, 31]. These frequencies are quite similar to the EXAFS findings, the Einstein frequency of the Fe-As to be about $225 \mathrm{~cm}^{-1}\left(\Theta_{E} \sim 324 \mathrm{~K}\right)$. The $\Theta_{E}$ for the Fe-As bonds are lower than the $\Theta_{E}$ for the Ru-As bonds. The $\Theta_{E}$ for the Ru-As bonds are found to be $353 \pm 42 \mathrm{~K}$ and $354 \pm 26 \mathrm{~K}$ respectively for the $x=0.25$ and $x=0.5$ samples. The $\sigma_{0}^{2}$ representing the random static disorder, is significantly higher for the $x=0.5$ sample, $\sim 0.0026 \AA^{2}$, compared to $\sim 0.0006 \AA^{2}$ for $x=0.0,0.05$ and 0.25 samples. Similar static disorder and force constants $\left(5.09,5.06\right.$ and $4.99 \mathrm{eV} / \AA^{2}$ respectively) for the Fe-As bonds in $x=0.0,0.05$ and 0.25 indicate that the atomic disorder in the FeAs layer may not have a direct effect the electronic transport. Indeed, the residual resistivity $\rho_{0}$ changes anomalously as a function of $\mathrm{Ru}$ concentration. The $\rho_{0}$ is $\sim 0.3$ $\mathrm{m} \Omega \mathrm{cm}$ for the pure $\mathrm{SmFeAsO} \mathrm{A}_{0.85} \mathrm{~F}_{0.15}$ system and increases sharply with the $\mathrm{Ru}$ substitution reaching a maximum value of about $2 \mathrm{~m} \Omega \mathrm{cm}$ for $x=0.25$ while the $\mathrm{T}_{c}$ decreases [21]. With further Ru substitution the $\rho_{0}$ decreases by half at $x=0.5\left(\rho_{0} \sim 2 \mathrm{~m} \Omega \mathrm{cm}\right)$ and has a similar value of $\rho_{0} \sim 0.3 \mathrm{~m} \Omega \mathrm{cm}$ for the $x=1$. Therefore, it appears that the impurity scattering from the substituted ruthenium being dominant to describe the transport phenomena for $x \leq 0.25$.

On the other hand, the larger static disorder of the Fe-As bonds in $x=0.5$ sample $\left(\sigma_{0}^{2} \sim 0.0026 \AA^{2}\right)$, albeit with the force constant being similar to the other samples $\left(4.83 \mathrm{eV} / \AA^{2}\right)$, suggests that some different mechanism should be active to describe the electronic transport, i.e. decreased $\rho_{0}$ and the $T_{c}$ for $x \geq 0.25$. In addition, the force constant for the Ru-As distance remains the same for $x=0.25$ and 0.5 samples, (6.04 and $6.07 \mathrm{eV} / \AA^{2}$, respectively). Therefore, it is likely that the title system is phase separated even at $x=0.25$, and the reduced $\rho_{0}$ from $x=0.25$ to $x=0.5$ is merely due to the increased density of states with increasing $\mathrm{Ru}$ because of more extended $\mathrm{Ru} 4 \mathrm{~d}$ states than Fe $3 \mathrm{~d}$, consistent with the density functional theory calculations [21]. It should be mentioned that the active and spacer layers are getting decoupled and thinner with the Ru substitution [19], i.e., electronically the system contains active FeAs layers which have poorer screening from the spacer layers, and hence can suffer phase separation as the case of ternary $\mathrm{FeSe}_{1-x} \mathrm{Te}_{x}$ [15]. Since the characteristic length scale of EXAFS is about a nanometer, it can be fairly argued that the observed phase separation is at a nanometer length scale, that is consistent with $\mu$ SR measurements $[22]$ on the similar system. It is also interesting to note that similar nanoscale textures have been observed in the Ru-substituted $\mathrm{BaFe}_{2} \mathrm{As}_{2}$ (122) system by NMR experiments due to inhomogeneous destruction of antiferromagnetic order by Ru substitution [32].

We can also notice that the $\sigma_{i}^{2}$ for the Ru-As bondlength manifests an upturn at a temperature $\sim 40 \mathrm{~K}(\mathrm{Fig}$. 4 ). This appears to be consistent with the anomaly observed in zero field $\mu \mathrm{SR}$ measurements, sensitive to short range 
magnetic order, revealing similar change in the Ru substituted 1111-system [22]. However, the observed change is very small to be stressed further and more experimental work is needed before it can be argued if the short range magnetic order is coupled to the charge and atomic displacements for particular Ru concentrations.

In summary, we have studied temperature dependent local structure of $\mathrm{SmFe}_{1-x} \mathrm{Ru}_{x} \mathrm{AsO}_{0.85} \mathrm{~F}_{0.15}$ system for varying $\mathrm{Ru}$ concentration by As $K$-edge EXAFS. We find that Ru substitution effect is mainly confined to the electronically active FeAs layer, and the Ru substituted system has different Fe-As and Ru-As distances. The force constants of the Fe-As and Ru-As bonds do not show any change with the Ru concentration, indicating coexisting electronic phases in the isoelectronic substitution. The static disorder in the Fe-As bonds remains unchanged as the bond strength for $x \leq$ 0.25 , suggesting that the transport properties of the system should be described mainly by the impurity scattering in FeAs layers. With further Ru substitution, the extended $\mathrm{Ru} 4 \mathrm{~d}$ states affects substantially the electronic density of states at the Fermi level, and hence the transport phenomena. On the basis of present results we can conclude that upon isoelectric substitution the title system breaks down into coexisting nanoscale electronic phases due to the frustration of interlayer atomic correlations.

\section{Acknowledgments}

The authors thank Sergey Nikitenko and Miguel Silveria of BM26A, ESRF, Grenoble and Luca Olivi, Giuliana Aquilanti and Nicola Novello, of XAFS, ELETTRA, Trieste, for their active cooperation in the EXAFS measurements.

[1] Celebrating 100 years of superconductivity - Special issue on Iron Based Superconductors: Report on Progress in Physics, volume 74, number 12 (2011) .

[2] D. C. Johnston, Advances in Physics 59, 803 (2010).

[3] D. Johrendt, H. Hosono, R.-D. Hoffmann, R. Pttgen, Zeitschrift fr Kristallographie - Crystalline Materials 226, 435 (2011); C.H. Lee, K. Kihou, A. Iyo, H. Kito, P.M. Shirage, H. Eisaki, Solid State Communications, 152, 644 (2012); Zhongxian Zhao, Xiaoli Dong, Liling Sun, Solid State Communications, 152660 (2011).

[4] Chul-Ho Lee, Akira Iyo, Hiroshi Eisaki, Hijiri Kito, Maria Teresa Fernandez-Diaz, Toshimitsu Ito, Kunihiro Kihou, Hirofumi Matsuhata, Markus Braden, and Kazuyoshi Yamada, Journal of the Physical Society of Japan 77, 083704 (2008).

[5] Kazuhiko Kuroki, Hidetomo Usui, Seiichiro Onari, Ryotaro Arita, and Hideo Aoki, Physical Review B 79, 224511 (2009); V. Vildosola, L. Pourovskii, R. Arita, S. Biermann, and A. Georges, Physical Review B 78, 064518 (2008).

[6] X-ray Absorption: Principles, Applications, Techniques of EXAFS, SEXAFS, XANES, edited by R. Prins and D. C. Koningsberger (Wiley, New York, 1988).

[7] N. L. Saini, A. Lanzara, H. Oyanagi, H. Yamaguchi, K. Oka, T. Ito, and A. Bianconi, Physical Review B 55 , 12759 (1997).

[8] N. L. Saini, H. Oyanagi, A. Lanzara, D. Di Castro, S. Agrestini, A. Bianconi, F. Nakamura, and T. Fujita, Physical Review B 64, 132510 (2001); N. L. Saini, A. Bianconi, and H. Oyanagi, Journal of the Physical Society of Japan 70, 2092 (2001).

[9] H. Oyanagi, A. Tsukada, M. Naito, and N. L. Saini, Physical Review B 75, 024511 (2007).

[10] A. Bianconi and N.L. Saini, Structure and Bonding 114, 287 (2005).

[11] C. J. Zhang, H. Oyanagi, Z. H. Sun, Y. Kamihara, and H. Hosono, Physical Review B 78, 214513 (2008).

[12] C. J. Zhang, H. Oyanagi, Z. H. Sun, Y. Kamihara, and H. Hosono, Physical Review B 81, 094516 (2010). 
[13] A. Iadecola, S. Agrestini, M. Filippi, L. Simonelli, M. Fratini, B. Joseph, D. Mahajan and N. L. Saini, Europhysics Letters 87, 26005 (2009).

[14] B. Joseph, A. Iadecola, M. Fratini, A. Bianconi, A. Marcelli and N.L. Saini, Journal of Physics: Condensed Matter 21, 432201 (2009); W. Xu, et al., Journal of Physics: Condensed Matter 22, 125701 (2010); W. Xu, et al., Europhysics Letters 90, 57001 (2010); A. Ricci, et al., Superconductor Science and Technology 23, 052003 (2010).

[15] B. Joseph, A. Iadecola, A. Puri, L. Simonelli, Y. Mizuguchi, Y. Takano, and N. L. Saini, Physical Review B 82, 020502 (2010).

[16] B. Joseph, A. Iadecola, L. Malavasi, N. L. Saini, Journal of Physics: Condensed Matter 23, 265701 (2011).

[17] A. Iadecola, B. Joseph, L. Simonelli, Y. Mizuguchi, Y. Takano, and N. L. Saini, Europhysics Letters 90, 67008 (2010); A. Iadecola, et al., Journal of Physics: Condensed Matter 23, 425701 (2011).

[18] A. Iadecola, B. Joseph, L. Simonelli, A. Puri, Y. Mizuguchi, H. Takeya, Y. Takano and N. L. Saini, Journal of Physics: Condensed Matter 24, 115701 (2012).

[19] A. Iadecola, B. Joseph, L. Simonelli, L. Maugeri, M. Fratini, A. Martinelli, A. Palenzona, M. Putti, and N.L. Saini, Physical Review B 85214530 (2012).

[20] S. Nikitenko, A. M. Beale, A. M. J. van der Eerden, S. D. M. Jacques, O. Leynaud, M. G. O'Brien, D. Detollenaere, R. Kaptein, B. M. Weckhuysen, and W. Bras, Journal of Synchrotron Radiation 15, 632 (2008).

[21] M. Tropeano, M. R. Cimberle, C. Ferdeghini, G. Lamura, A. Martinelli, A. Palenzona, I. Pallecchi, A. Sala, I. Sheikin, F. Bernardini, et al., Physical Review B 81, 184504 (2010).

[22] S. Sanna, P. Carretta, P. Bonf, G. Prando, G. Allodi, R. De Renzi, T. Shiroka, G. Lamura, A. Martinelli, and M. Putti, Physical Review Letters 107, 227003 (2011).

[23] A. Martinelli, M. Ferretti, P. Manfrinetti, A. Palenzona, M. Tropeano, M. R. Cimberle, C. Ferdeghini, R. Valle, C. Bernini, M. Putti, A. S. Siri, Superconductor Science and Technology 21, 095017 (2008).

[24] D. H. Ryan, J. M. Cadogan, C. Ritter, F. Canepa, A. Palenzona, and M. Putti, Physical Review B 80, 220503 (2009).

[25] J. Mustre de Leon, J. J. Rehr, S. I. Zabinsky, and R. C. Albers, Physical Review B 44, 4146 (1991); J. J. Rehr and R. C. Albers, Reviews of Modern Physics 72, 621 (2000).

[26] Error reporting recommendations, International XAS Society, Standards and Criteria Commitee, 2000 (http://ixs.csrri.iit.edu/IXS/).

[27] R.D. Heyding, L.D. Calvert, Canadian Journal of Chemistry 35, 449 (1957); R.D. Heyding, L.D. Calvert, Canadian Journal of Chemistry 39, 955 (1961).

[28] E. Sevillano, H. Meuth, J.J. Rehr, Phys. Rev. B20, 4908 (1979).

[29] see, e.g. a review by J. J. Rehr and R. C. Albers, Reviews of Modern Physics 72, 621 (2000).

[30] C. Marini, C. Mirri, G. Profeta, S. Lupi, D. Di Castro, R. Sopracase, P. Postorino, P. Calvani, A. Perucchi, S. Massidda, G. M. Tropeano, M. Putti, A. Martinelli, A. Palenzona and P. Dore, Europhysics Letters 84, 67013 (2008).

[31] V. G. Hadjiev, M. N. Iliev, K. Sasmal, Y.-Y. Sun, and C. W. Chu, Physical Review B 77, 220505R (2008).

[32] Y. Laplace, J. Bobroff, V. Brouet, G. Collin, F. Rullier-Albenque, D. Colson, and A. Forget, Physical Review B 86, 020510 (2012). 